\title{
Cosmic rays in the Orion-Eridanus superbubble
}

\author{
Theo Joubaud, Isabelle Grenier, Jean-Marc Casandjian \\ Dap/AIM/CEA Saclay, Universite Paris Diderot, France \\ E-mail: theo.joubauddcea.fr
}

\begin{abstract}
The Orion-Eridanus superbubble, formed from the winds and the explosions of Orion's massive stars, could be a cosmic-ray acceleration site. Inside the superbubble, the large level of magnetohydrodynamics turbulence and the core-collapse supernovae have created a turbulent medium which effect on cosmic rays can be probed comparing their flux and spectrum in the superbubble to the average in nearby interstellar clouds.

To study cosmic rays in the superbubble, we first rely on Fermi Large Area Telescope data. Eight years of data and gamma rays above $250 \mathrm{MeV}$ have been used. We are particularly interested in gamma rays resulting from the decay of neutral pions produced by the interaction of cosmic rays with interstellar gas. Hence, knowing both gas distribution and gamma-ray emission allows to obtain the cosmic-ray flux. We thus developed a model of the interstellar emission using multiwavelength tracers for the gas column densities in the different phases (atomic, molecular, ionized) of the superbubble in which atomic and molecular phases are further divided into several complexes based on coherence in spatial distribution and velocity. The model also includes other ancillary components such as inverse-Compton emission and point sources.

Preliminary results show that the emissivity spectrum of the main HI cloud is consistent with the average spectrum measured in nearby clouds located outside the superbubble, but within the Gould Belt. This uniformity calls for a detailed assessment of the recent supernova rate and the energetics of massive stellar winds in the superbubble in order to estimate the diffusion properties of the young cosmic rays and to evaluate the need, or not, to advect them away in the Gould Belt or to the halo via the local Galactic wind.
\end{abstract}

7th Fermi Symposium 2017

15-20 October 2017

Garmisch-Partenkirchen, Germany 